\title{
Fostering Equitable Help-Seeking for K-3 Students in Low Income and Rural Contexts
}

\author{
Judith, O, Uchidiuno \\ Human Computer Interaction Institute, Carnegie Mellon \\ University, Pittsburgh, USA \\ jio@andrew.cmu.edu \\ Jessica, Hammer \\ Human Computer Interaction Institute, Carnegie Mellon \\ University, Pittsburgh, USA \\ hammerj@cs.cmu.edu
}

\begin{abstract}
Adaptive Collaborative Learning Support (ACLS) systems improve collaboration and learning for students over individual work or collaboration with non-adaptive support. However, many ACLS systems are ill-suited for rural contexts where students often need multiple kinds of support to complete tasks, may speak languages unsupported by the system, and require more than pre-assigned tutor-tutee student pairs for more equitable learning. We designed an intervention that fosters more equitable help-seeking by automatically detecting student struggles and prompts them to seek help from specific peers that can help. We conducted a mixed-methods experimental study with $98 \mathrm{~K}-3$ students in a rural village in Tanzania over a one-month period, evaluating how the system affects student interactions, system engagement, and student learning. Our intervention increased student interactions by almost 4 times compared to the control condition, increased domain knowledge interactions, and propelled students to engage in more cognitively challenging activities.
\end{abstract}

\section{CCS CONCEPTS}

- Applied computing $\rightarrow$ Education; Computer-assisted instruction.

ACM Reference Format:

Judith, O, Uchidiuno, Kenneth, R, Koedinger, Jessica, Hammer, and Amy, E, Ogan. 2021. Fostering Equitable Help-Seeking for K-3 Students in Low Income and Rural Contexts. In CHI Conference on Human Factors in Computing Systems (CHI '21), May 08-13, 2021, Yokohama, Japan. ACM, New York, NY, USA, 14 pages. https://doi.org/10.1145/3411764.3445144

\section{INTRODUCTION}

Help-seeking and peer-tutoring systems, such as Adaptive Collaborative Learning Support (ACLS) systems, have shown tremendous benefits for student learning, collaboration, group formation, and supporting domain knowledge acquisition (see e.g.,

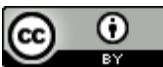

This work is licensed under a Creative Commons Attribution International 4.0 License.

CHI '21, May 08-13, 2021, Yokohama, Japan

(C) 2021 Copyright held by the owner/author(s)

ACM ISBN 978-1-4503-8096-6/21/05.

https://doi.org/10.1145/3411764.3445144

\author{
Kenneth, R, Koedinger \\ Human Computer Interaction Institute, Carnegie Mellon \\ University, Pittsburgh, USA \\ kk1u@andrew.cmu.edu \\ Amy, E, Ogan \\ Human Computer Interaction Institute, Carnegie Mellon \\ University, Pittsburgh, USA \\ aeo@andrew.cmu.edu
}

$[4,25,50,67,68])$. The benefits of peer-peer collaboration and help-seeking in the learning process includes increased cognitive activity [19], increased motivation and satisfaction [66], increased enthusiasm for learning over individualized learning settings [38], and higher literacy scores [18]. Rural villages are an appropriate setting to investigate the potential benefits of such peer-peer helpseeking systems for understudied populations. We focus our study in rural Tanzania, where several of the authors have been engaged in research for the past decade. Located in East Africa, Tanzania has a population of about 58 million people, with $80 \%$ living in rural areas and $44 \%$ less than 15 years old ${ }^{1}$. With such a high population of young people, the Tanzanian government has been focused on providing adequate education, from pre-primary to secondary school, to prepare students to be gainfully employed. In conjunction with other types of improvements to the education system, there is an opportunity for collaborative educational interventions to supplement traditional classroom instruction, with the expectation that peers could greatly benefit from scaffolded opportunities to support one another.

One requirement for ACLS systems is that they take a stance on how to group students who will support each other. Based on findings that high-performing students model desirable learning behaviors and help keep their lower-achieving peers on task, e.g. [22], one might assume that only the highest achieving students be selected as peer tutors. However, other studies provide evidence that high and low-performing students equally benefit from being assigned the role of a peer tutor $[15,31,70]$, especially when such interactions are adequately scaffolded $[8,39]$. Systems such as $[59,68]$ tend to assign selected heterogenous student pairs or groups to help one another, and the system intervenes to resolve conflict, improve collaboration and the quality of student interactions. Such systems may not consider the cultural and social dynamics that students experience and may exacerbate existing norms of unequal distribution of help-giving responsibilities in the classroom. Insights from our previous studies in this cultural context [65] show that lowerachieving students rarely get opportunities to provide support for their peers in the classroom. We compared the interactions between undesignated and designated helpers within groups. We found that one-sided expectations of helping that students experienced in their classrooms were reinforced, where academically weaker students were never called on to be helpers. Within existing social dynamics,

$\overline{{ }^{1} \mathrm{http}: / / \text { worldpopulationreview.com/countries/tanzania-population/ }}$ 
lower achieving students were likely to relegate themselves to the position of the tutee in assigned pair situations even when they were explicitly designated as tutors.

Therefore, we build upon our prior work by augmenting a K-3 learning system with a help-seeking system to investigate how to create equitable help-seeking conditions in group learning settings, rather than the pre-assigned tutor-tutee student pairs present in many ACLS systems. This system was designed to foster more equitably-distributed helping interactions in rural Tanzanian classrooms, regardless of students' domain knowledge. The rural context of our study comprised a population of students with varied digital literacy, who may need assistance with navigating a technological device or learning about the mechanics of learning applications [64]; this presents an opportunity for students to practice being a helper in group learning settings regardless of domain mastery. Specifically, our system was designed to support students' digital literacy needs and scaffold application navigation, in addition to the typical support for domain knowledge. Using a gesture recognition and performance monitoring algorithm, the system detects when students do not have adequate digital literacy or application navigation skills - or domain knowledge - to engage with a learning application. Struggling students are then directed to peers who can provide them adequate support with that particular skill. Direction to a helper selected randomly from amongst the set of helpers qualified on that particular skill ensures that every helper for a given problem can be called upon with equal probability. The confidence that students with low domain knowledge build providing effective support to their higher-achieving peers on non-domain skills may potentially build their confidence in other domain-related content areas and promotes more balanced helping interactions in learning groups.

Our research study was executed as a mixed-methods randomized controlled trial conducted over a one-month period where groups of students were provided with individual tablets preloaded with the learning system. We collected data from 98 K-3 students over 137 study sessions. The experimental groups were provided with the learning system augmented with a help-seeking system intervention, while the control groups were given the baseline learning system. There were no adults present during the study sessions, and all students were instructed to interact with one another as they pleased.

We investigated the following hypotheses for the experimental study:

H1 - Student Interactions:

a) Students in the experimental group will have significantly increased interactions with non-adjacent peers compared to the control group

H2 - System Engagement:

a) Students in the experimental group will have higher activity completion rates compared to the control group

b) Students in the experimental group will have significantly increased selections of activities that are perceived to be more difficult compared to the control group.

H3 - Learning: a) Students in the experimental group will have a significantly higher number of activities mastered within the learning system compared to the control group

Overall, this research study contributes to the scientific literature on designing ACLS systems specifically targeted for low infrastructure and diverse language contexts. We also contribute insights on the effectiveness of such help-seeking interventions in rural contexts where the presence of knowledgeable adults is not guaranteed.

\section{BACKGROUND}

\subsection{Peer Support and Tutoring in Learning Environments}

It is widely accepted in the learning sciences community that collaborative peer learning is beneficial for student learning outcomes. Collaborative learning is defined as "an instructional strategy in which students work actively and purposefully in small groups to enhance both their own and their teammates' learning" [1, 47]. Studies show that students learn more effectively when they learn in groups rather than working alone [13, 53]. Peers engage in instructional discourse that can bring about positive learning gains, including "modelling, assisting, directing, tutoring, negotiating, affirming, and contradicting each other", among others [55]. They engage in a variety of activities including lecturing, providing and evaluating answers, offering advice and problem-solving strategies, and motivating their peers [8]. However, studies also show that not all students are equally as likely to provide high-quality instructional discourse [3]. Prior research suggests that the quality of collaborative discourse is highly dependent on individual factors such as students' prior knowledge and self-efficacy, as well as their relationship to one another [40].

While some interventions pre-assign tutor/tutee pairs, as long as students are grouped according to similar age and skills, it has been shown that they can provide mutual support for one another with adequate structure [23]. Allowing children of similar age/skills the opportunity to support one another without pre-assigned roles promotes a more equitable model of help-seeking and help-giving. Across different cultures, high academic achievement is positively associated with peer acceptance [9], therefore, pre-designating some students to only receive help might have negative effects on their social status and interaction with their peers.

The support that students offer one another becomes even more important when educational technologies are deployed in unsupervised settings, where children must rely on each other to navigate and learn from such systems. Some research studies have reported positive outcomes from unsupervised settings, reporting an increase in student curiosity [32], and even marginal learning gains [6], but do not provide any insights on the social interactions that lead to such outcomes. For instance, Kumar et al. provide rich insights from conducting an unsupervised learning research study using a mobile phone in rural parts of India. They found that beyond infrastructural issues such as inconsistent electricity, factors such as gender, caste, and time of day significantly affected student interactions with the learning content. The authors report that children learned to support their peers from watching the experimenters navigate the devices but provide few details on how this knowledge 
transfer occurred. As Pea, among others have pointed out, while intelligence may be distributed among children learning together and the tools they use to learn, such learning can benefit from guidance and structure from adults as well as from the intentional design of learning technologies to support collaborative behaviors $[43,44]$.

\subsection{Cultural Factors that Influence Help-Seeking and Help-Giving Behaviors}

Cultural factors influence how people demonstrate their mastery of knowledge; ignoring these practices can cause undue stress and demotivate students. Teaching practices that disturb these accepted patterns of student interactions (such as promoting peer-peer helpseeking) may be detrimental to the students' psychological wellbeing. One example is the debate style of discourse encouraged in many Western classrooms - while this can provide to one form of peer learning, it may feel uncomfortable for students from cultures where high power distance is a norm, or for students who value group harmony over asserting one's individuality. Students from some cultures have been found to feel more comfortable questioning the teacher, even without mastery of the subject, while students with other cultural influences may prefer initially receiving direct instruction and avoid participation until they have achieved mastery [72]. The desire to promote harmony may cause students who identify with collectivist cultures discomfort when forced to publicly express their opinions - students may also feel apprehensive of making mistakes or making others feel uncomfortable [11, 6062]. Culturally-ascribed gender roles can also intersect with group dynamics, affecting classroom interactions. In some societies, for instance, males have more influence in group discussions, and have a greater chance of being elected group leader [46]. In Tanzania specifically, research studies suggest that there are clearly ascribed differences in the expectations and responsibilities that girls are given compared to boys [29].

Outside of the classroom, the everyday living practices of many Tanzanians has been found to be reflective of a culture where helpgiving and collaboration is the norm. A qualitative study by Evans [17] reports that in Tanzanian households, older siblings generally have the responsibility of instructing, guiding, looking after, making money, and doing homework with their younger siblings to free up their parents for other income earning and family care activities. It is common practice for neighbors and friends to help children cook and care for their sick siblings to allow them go to school [26] Neighbors may give children the opportunity to earn money for their school fees in exchange for doing light domestic tasks [37]. Nalkur et al. [37] report that children experiencing homelessness in Tanzania, with extremely limited resources, feel an overwhelming sense of responsibility towards other children. Since help-seeking and help-giving are so prevalent for many groups in Tanzania, educational technologies designed to foster such behaviors may especially thrive as it allows students to engage in behaviors that they routinely engage in outside school contexts - although it may conflict with the practices of in-school authority figures such as teachers.

\subsection{Rural Education in Tanzania}

Access to education is considered a basic human right for every child in Tanzania and the government has made directed efforts at improving classroom infrastructure, and teacher recruitment [57]. These efforts have led to increased access to education, but do not include specific guidelines on important factors such as teacherstudent ratios, classroom sizes, or physical infrastructure. As expected, such loose guidelines disproportionately impact schools in rural areas where classrooms often have insufficient desks, and classroom sizes reach close to 100 children $[34,35]$. This places a higher burden on teachers to spend valuable time managing classroom dynamics and makes individual attention to students difficult.

Also, there are challenges on the demand for education - the opportunity cost for parents to enroll their children in school rather than engage in other short-term economically productive activities is high [27], the rigid schedule of formal schooling does not take agricultural harvest seasons into account, and the perceived lack of alignment between school content and local contexts reinforce ideas that school was created for those from a different culture [58]. Even when rural parents place a high value on schooling, their own lack of education may limit their ability to discuss academic topics with their children [58]. Unfortunately, these trends have not improved over the years - reports show that the number of out-of-school children in Tanzania had almost doubled in 2017, and that while pre-primary registration numbers are increasing, enrollment in other levels of education have been decreasing over the years ${ }^{2}$.

Finally, Tanzania faces major challenges with recruiting teachers to work in rural schools. Lack of adequate transportation facilities makes everyday activities such as getting to schools, doctor and family visits, collecting their salaries etc. very difficult for teachers [24, 36]. A study conducted in 2013 showed that about an average of $24 \%$ of Tanzanian teachers (in rural and urban areas) were absent at the time of an administered survey [74]. To alleviate issues related to teacher recruitment, neighboring countries offer attractive monetary incentives to teachers who elect to teach in rural areas. Specific regions in Tanzania have instituted incentives (such as set salaries, bicycles for transportation, etc.) to attract teachers to their regions [36, 57]. However, studies showed that these policies result in no significant increases in workers' retention in the ministry of education [28]; teachers tended to leave for urban cities after mandatory working years were complete [10].

All these factors, along with the limited opportunity for professional development for teachers in rural areas, increase the educational disparities in Tanzanian rural areas. There is currently a healthy debate over the extent to which technology introduces new issues, such as maintaining existing cultural norms for teaching and learning, and unnecessary expenses for governments. On the other side of the debate, peer-peer support opportunities facilitated by education technologies have been demonstrated to be effective in extensive investigations with Western populations and, this work is a step towards understanding their application with understudied learners.

\footnotetext{
${ }^{2}$ http://uis.unesco.org/country/TZ
} 


\section{METHODOLOGY}

\subsection{System Design}

The learning application used in this study was focused on the following learning areas, deployed in Swahili: literacy (letter and phonemic awareness, writing, stories curated from the African Storybook Project ${ }^{3}$, and math (number identification, number writing, addition and subtraction). The system's main menu allowed students to select from 'Math', 'Reading and Writing', and 'Stories'. While "Reading and Writing" and "Stories" both fall under the literacy domain, "Stories" is equivalent to an audio book library where students could listen to stories passively without the pressure of assessments. All students had access to all content areas. The treatment and control groups were provided the exact same learning system on personal tablets, however students in the treatment group had the help-seeking intervention enabled in their system on top of the content. To power the help-seeking intervention, the pre-test and baseline sessions provided us with information on students' initial knowledge levels and activities they had successfully navigated. This allowed the system to reliably suggest a helper who could provide adequate support. We updated this helper information daily as students mastered more knowledge components and encountered more activities.

Our prior research with this population $[64,65]$ found that students needed the following kinds of support to interact with tabletbased learning technologies: gesture support, application support, and domain knowledge support. Our help-seeking intervention was designed to automatically detect when students were struggling with these three error types, and an intervention was triggered after repeated struggle. Gesture errors were triggered when students continuously performed incorrect gestures in an application (e.g. swiping instead of tapping). Application errors were triggered when students did not correctly interact with applications e.g. a student is tapping correctly but on the wrong target. Finally, domain knowledge errors were triggered when students failed to achieve mastery in an activity (below $70 \%$ passing score).

Our baseline learning system already adjusts students' activity levels based on student performance to ensure that they are placed in cognitively appropriate activities. Therefore, we expected to detect much fewer occurrences of domain knowledge issues compared to the application level issues. Both "Digital Literacy Support" and "Application Support" required that a gesture recognition algorithm was constantly running in the background. This was implemented using the Android GestureDetector library which provides native support for detecting the following gestures: tap, double tap, long press, fling (or swipe), and a customizable motion listener. When errors were detected, the system suggested that students call on specific peers for assistance. The help-seeking intervention was pre-populated with information related to all students in a group including their existing domain knowledge, experience with applications, and their picture. When triggered, students heard the following message on the system intervention: "If you need help solving this problem, call on this friend [here a picture of the other student was shown]. They will be happy to teach you so you can teach others. Touch this picture to continue". Student helpers were

\footnotetext{
${ }^{3}$ http://www.africanstorybook.org/
}

selected based on those who had successfully mastered a domain area or completed an activity. Based on their ongoing interactions in the system during the intervention period, student performance information and their application experience was updated daily to increase the probability of every student getting called upon appropriately for help. Figure 1 shows an example of the system intervention triggered and the help-seeking suggestion.

The control condition interacted with the baseline learning system without the help-seeking intervention enabled.

\subsection{Study Design}

We conducted a between subjects, repeated measures experimental study with 98 (girls $=50$; boys $=48)$ primary school students ages $5-10$ in a rural village in Tanzania with which the research team has had a partnership for a number of years. The students were distributed across 5 classes - Nursery 1, Nursery 2, Primary 1, Primary 2, and Primary 3. The students in Nursery 2 - Primary 2 had prior exposure to the learning technology from a previous study $[64,65]$. Each experimental session lasted about 1 hour, and we collected data from 137 (68 control and 69 intervention) total sessions across all grades. Non-adjacent peers indicate learners who do not sit directly beside the focal learner. Students were seated on a long rectangular table that could seat 6 children with adequate spacing.

Since student prior relationships are an important factor to consider in any intervention that involves student interactions, the study groups were created to account for existing friendships within each classroom. Prior research studies on group learning and motivation show that performance is linked to increased diversity peer relationships, gender and age differences, and personality traits $[2,41,52]$. A technique called Sociometry was used to determine the inter-relationships within a group. Its purpose is to discover group structure: i.e., the basic "network" of friendship patterns and sub-group organization [51]. A cohesion score [51] is a numerical measure that shows the relationship strength within a group. This sociometry approach was critical to creating the treatment groups rather than using a pure random assignment because existing social dynamics can explain whether students agree to seek, or offer help to specific peers [48]. Not accounting for prior friendships in the group formation would have presented a confound to the effects that the intervention had on student interactions. As a result, other pre-experiment attributes such as pre-test scores were not random error and were included as covariates in our regression models.

Prior to the first day of the study, each student was presented with a set of pictures of all the students in their classroom and asked to identify the students they liked to play with. After this data was collected, randomized groups of 5-6 students were created and group cohesion scores were calculated within each grade. This allowed for all students to have at least one non-adjacent peer. Groups were then re-randomized to ensure that each group had an equivalent cohesion score, and then randomly assigned to either a treatment or control condition. Figure 2 shows an example friendship network map of all the students in one class, and how the randomized groups were created. In all grades, girls only selected other girls as their friends, and boys did the same as well. There were no grades with cross gender friendships indicated by 


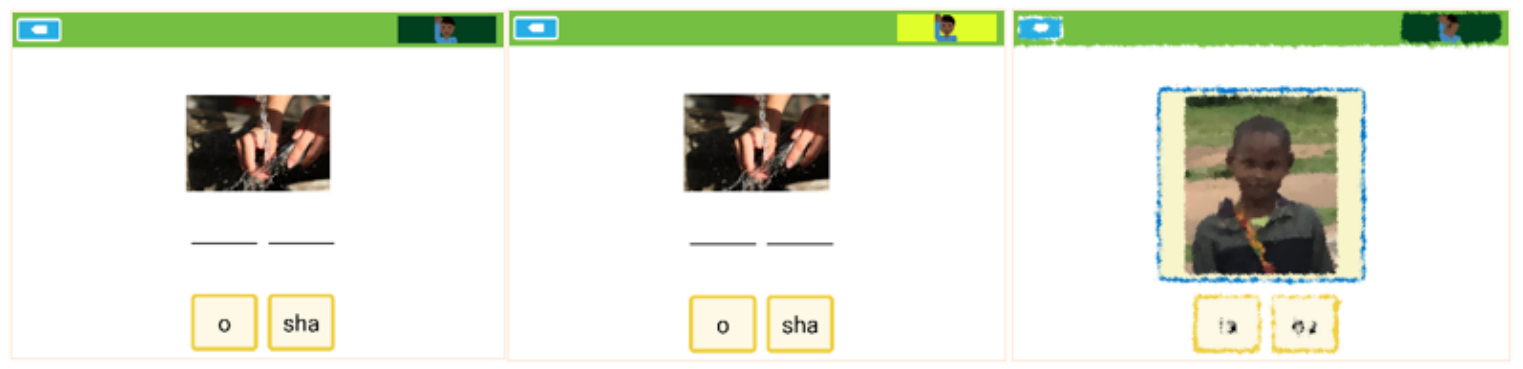

Figure 1: (L-R) Example of normal activity, help-seeking alert, and proposed intervention

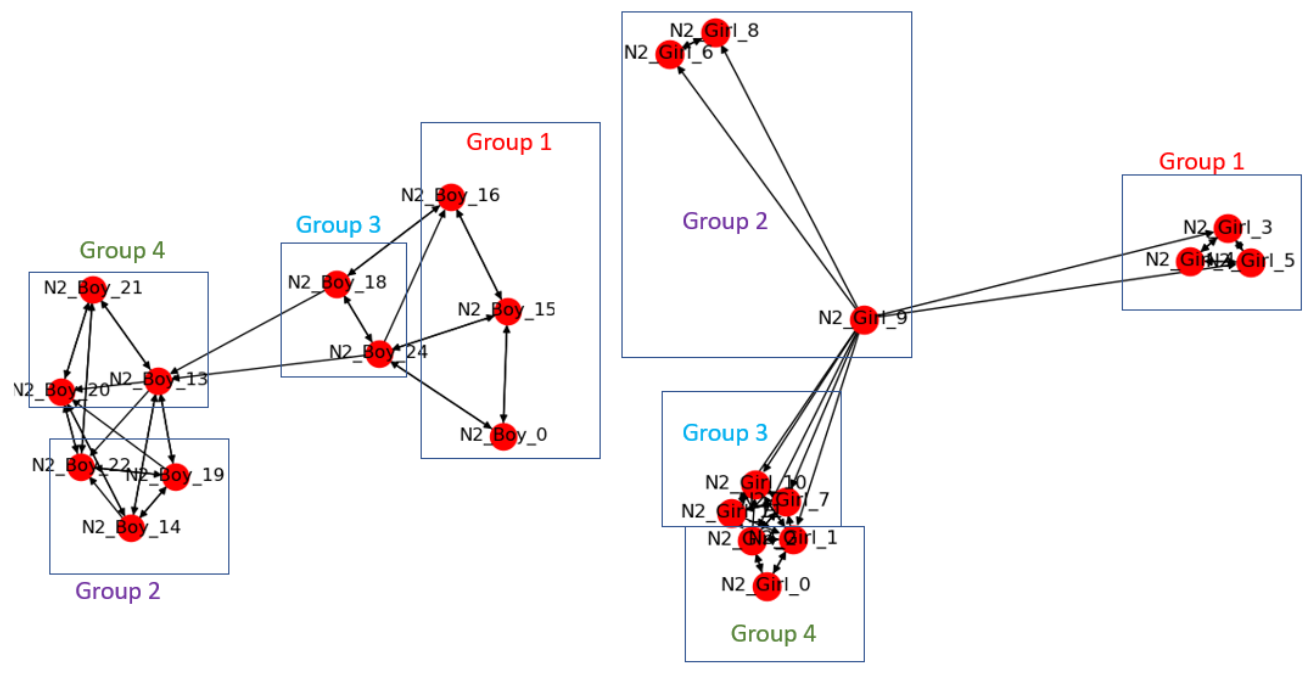

Figure 2: Nursery 2 Friendship Networks and Experiment Groupings

the students. Altogether, 18 groups of students were formed with 9 groups in each condition.

Each group was observed for 7 - 8 sessions throughout the study. This number included one baseline and one end line assessment session in which students used the learning system without the help-seeking intervention enabled. Students in all groups were informed that they were allowed to interact and collaborate with one another as they pleased. No researchers or adults were present in the room with the students during the study sessions. Instead, these unsupervised sessions were video recorded from multiple angles to capture natural student interactions. Written consent was obtained from all parents, the school authorities, and the village council.

\subsection{Data Collection and Analysis}

We reviewed videos from all group sessions and recorded qualitative observations of the student interactions with the tablet and one another. We were especially interested in non-adjacent student interactions, as our previous studies showed that students primarily engaged with their adjacent peers and ignored other students in the group. By adopting a partial-interval recording method [21], we broke each video session into 30 second intervals and for each child we annotated the videos to indicate: 1) the time of the interaction, 2) the helping and receiving student, as well as 3) the type of help the student was providing e.g. application support, gesture or technical support, application support, domain knowledge support, or general information sharing. We then reviewed the video in conjunction with the system logs to annotate when 1) the system recommended a helper to a student, 2) the type of error detected automatically, 3 ) if the student visibly appeared to need help, 4) the type of help they were likely to have needed, 5) whether the student activated the helper button, and 6) whether they followed through with the help suggestion by calling on the suggested student for help. We additionally annotated across all videos and all students for any instances where it appeared that a student needed help but the system did not intervene.

Three members of the team reviewed the videos, notes, and observation logs to identify emergent themes related to help-seeking and help-giving behaviors, as well as student interactions with the system following a grounded theory approach [12]. Every week, these annotations were reviewed with supervising members of the team who provided guidance on areas that needed further investigation. In the experimental conditions, the qualitative annotations were triangulated with the quantitative logs gathered by the system intervention on when each intervention was triggered, and the helper suggested. After all sessions were annotated, all three 


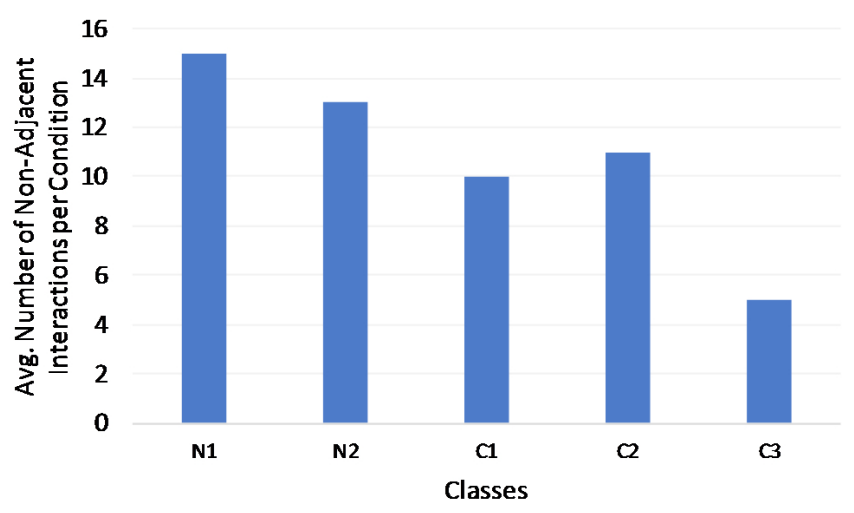

Figure 3: Number of Baseline Non-Adjacent Interactions by Class

members of the team reviewed the findings, discussed all areas of disagreement, and re-categorized findings as agreed upon by the entire team. Where necessary, we consulted with native Swahili speakers to help translate interactions, as well as provide insights on the cultural underpinnings of those interactions. This study design and data analysis methodology has been used and validated in previous learning science research studies e.g. [42].

Quantitatively, we began our data analysis by running basic descriptive statistics to gain high level insights on student performance and system interactions. These high-level statistics provided direction to properly annotate, triangulate, and perform detailed analysis on the video data. Finally, we performed hierarchical linear regressions and independent $t$-tests to measure the effects of the helper system on student performance between the experimental and control groups.

\section{FINDINGS}

For the sake of language consistency, the following grades (classes) are denoted by their short forms: Nursery 1 (N1), Nursery 2 (N2), Primary/Class 1 (C1), Primary/Class 2 (C2), and Primary/Class 3 (C3). Students who provide help will be referred to as "helpers", while those who receive help will be called "beneficiaries". Finally, 'treatment" group or experimental condition (vs control) will be used to refer to students who received our help-seeking intervention.

\subsection{Initial Student Reactions And Adjustments To Help-Seeking Intervention}

To begin our experiment sessions, we conducted baseline technology learning sessions with all 18 groups of students. Generally, the older students were, the lower the number of non-adjacent interactions they had with one another (Figure 3). This trend was not dependent on prior exposure to technology - students in both N1 and $\mathrm{C} 3 \mathrm{had}$ no prior exposure to our learning software before the baseline session yet interacted very differently with one another and the technology.

Once the help-seeking intervention was introduced after the baseline (treatment session 1), students had varying reactions to it.
The youngest students (N1) noticed the alerts going off but never actually activated it until the fourth session when one student activated it and showed it to a peer. Although N1 students were very excited to see their faces on the tablets, they never called their peers for help throughout the entire experiment despite being instructed to do so every session. Previous studies show that students begin to independently plan and collaborate starting from age five [69] given that the youngest students in N1 were 5 years old, and it was the beginning of the school year, they may have been too young to utilize the intervention.

Students in the other grades noticed and activated the intervention almost immediately and were also very excited to see their faces on the tablet. However, some never actually called their peers for help despite the system suggestions. Others called for help but their peers walked over to them and dismissed the intervention prompt, without helping, especially during the first two experimental sessions. There are several possible explanations for this behavior. First, while we instructed students to call on their friends for help, it was possible that helpers did not understand what that meant in practice which caused them to dismiss the intervention without actually helping. Also, our system highly relied on students listening to audio prompts to get placed in cognitively appropriate content, as well as follow the intervention instructions. Data from the recorded videos shows students bringing tablets up to their ears to hear instructions due to background noise from the other tablets and surrounding classrooms. As a result, most students got placed in content areas that were too easy for them, therefore did not need any help with the learning materials. To adjust for these difficulties, we instructed students to sit and work together on activities when called upon after session 3, and had a few students act out appropriate ways to help at the beginning of every session. We also adjusted our system to place all students in grade appropriate content areas starting from session 3 to reduce the dependence on sound for the knowledge placement tests.

On the design of the help-seeking intervention, students recognized the helper icon and understood that its purpose was to allow them digitally raise their hands - like they did in their classrooms (Figure 4). We observed several incidents of students struggling with an activity and repeatedly tapping the intervention for help. 


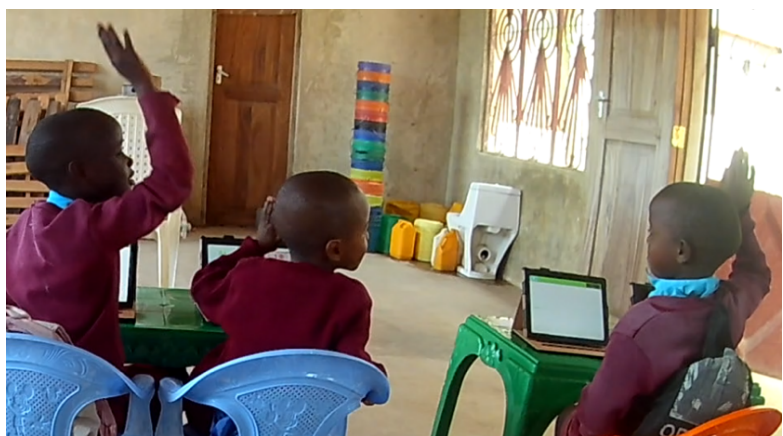

Figure 4: Students recognize the helper icon as a way to digitally raise their hands

We also observed students redirecting their adjacent peers to tap on the helper icon if the peer needed help that they could not provide. Like our previous studies [65], we found that students who were called upon were quite persistent in their help-giving, working together until the student could navigate the activity unassisted. Students also sometimes used the help requests as opportunities to practice activities that they had not encountered on their tablets interacting even after the help request was fulfilled.

\subsection{H-1: Interaction with Non-Adjacent Peers}

4.2.1 The Intervention Elicits Much Higher Movement From NonAdjacent Students. Students called on their peers much more overall in the treatment condition especially in the earlier sessions resulting in a higher number of non-adjacent student interactions. On the average, our system recommended students in the bottom pretest score quartile 1.85 times, second quartile 2.15 times, 3rd quartile 2.67 times, and top quartile 3.52 times per session. This shows that even the lowest performing students got some opportunities to practice providing support for their higher achieving peers. Figure 5 shows the average number of non-adjacent interactions in the control group sessions compared to the treatment groups.
The intervention did not alter normal student-student interactions; like the control groups, students in the treatment groups called on each other for different reasons even without prompting by the intervention. However, the intervention caused students to request for additional help, over what they would have requested unprompted.

The most prevalent type of interaction observed in the control condition was information sharing - this was when students called on their friends to share a new activity or read a new story together. In the earlier sessions, students called on their peers more for technical support which mostly happened when their tablets were switched off and they needed help getting back in. In the treatment condition, approximately $97 \%$ of the support calls triggered by the intervention was for application and gesture support, and $3 \%$ was for knowledge support. We did not observe any calls for knowledge support in the control groups. The requests for application support requests in the treatment condition was mostly observed in the $\mathrm{N} 1$ and $\mathrm{C} 3$ grades - this was unsurprising because unlike other grades, they had no prior experience with the technology.

We ran a hierarchical linear regression model, including the student classes (to account for effects of student ages), and the session number (to account for repeated exposure) to the model (Table 1). Students in the treatment groups interacted with each other almost three times more than the control groups. These results confirm our hypothesis $\mathrm{H} 1$ - the intervention causes students to interact at a higher frequency with their peers. Overall, students in higher grades interacted less with one another - students also interacted less as they gained more experience with the system.

Prior friendships did not affect students help-seeking requests or responses to the intervention. Throughout our analysis of the data, we qualitatively annotated if students behaved differently if the intervention recommended that they call on their friends for help. We found that students followed the system help recommendations regardless of whether their friends were suggested or not.

4.2.2 The Intervention Fosters Knowledge Support Interactions, And Students Get Better At Monitoring Their Cognition Over Time. The

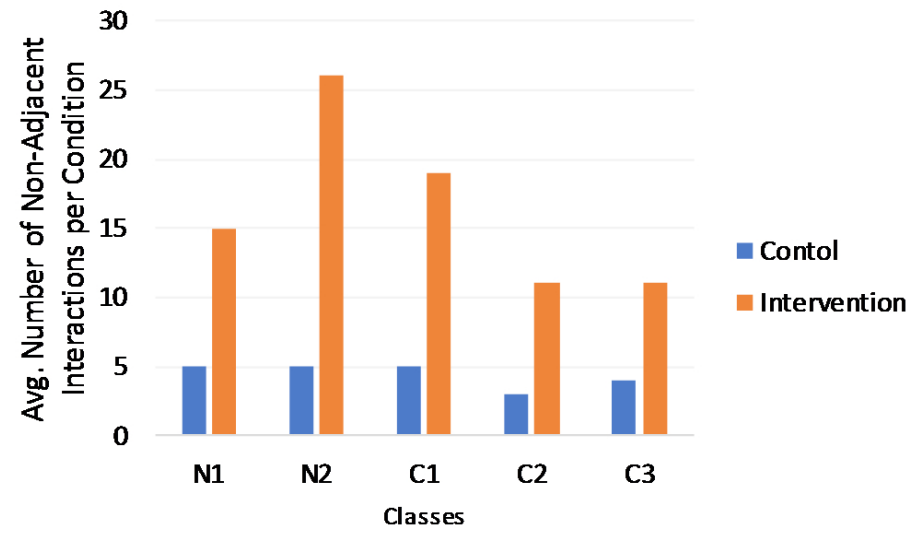

Figure 5: Average Number of Non-Adjacent Student Interactions in the Control vs Intervention (treatment) Conditions 
Table 1: Hierarchical Linear Regression model on the effects of the intervention, student grades (to account for effects of student ages), and the session number (to account for repeated exposure) on the Number of Non-Adjacent Peer Interactions (outcome variable)

\begin{tabular}{llll}
\hline & Estimate & Std. Error & $\mathrm{t}$ value \\
\hline Class - N1 Control Group (Intercept) & 4.46 & 4.76 & 0.94 \\
System Intervention & 11.13 & 2.35 & 4.73 \\
Class - N2 & 5.38 & 4.32 & 1.25 \\
Class - C1 & 0.94 & 4.36 & 0.22 \\
Class - C2 & -2.56 & -0.61 \\
Class - C3 & -3.12 & -0.71 \\
Session Number - 1 & 11.00 & 2.40 \\
Session Number - 2 & 0.06 & 4.39 & \\
Session Number - 3 & -4.67 & 4.58 & -1.01 \\
Session Number - 4 & 4.06 & 4.58 & \\
Session Number - 5 & -0.86 & 4.58 & \\
Session Number - 6 & -3.07 & 4.73 & \\
\end{tabular}

Table 2: Total Number of Help Requests by Support Type in Control and Treatment Conditions

\begin{tabular}{llllll}
\hline & ApplicationSupport & TechnicalSupport & InformationSharing & KnowledgeSupport & $\begin{array}{l}\text { Average calls for request per } \\
\text { session }\end{array}$ \\
\hline Control & 13 & 82 & 224 & 0 & 4.7 \\
Treatment & 634 & 165 & 223 & 29 & 15.5 \\
\hline
\end{tabular}

previous findings section was focused on the quantity of student interactions as a result of the intervention - this section focuses primarily on the quality of the help requests. In all 69 treatment sessions observed, there was a total of twenty-nine calls for knowledge support. Table 2 shows the total number of help requests by support type in both the control and treatment conditions. Our learning system automatically adjusts content presented based on student performance, so we were not surprised by the few number of requests for knowledge support. However, in sessions where knowledge support interactions were observed, students demonstrated they could support their peers appropriately.

In line with our previous findings and related literature, helpers mostly answered questions for their peers without any explanations. However, we observed a few knowledge building (explanations) interactions as a result of the intervention. In one session in C2 - a boy was struggling to complete an activity that required him to spell a word using its syllables. As a result, the intervention alerted him to call a girl for help and he did. She came over and answered the question for him, then went back to her seat. However, he continued to struggle with that activity and shortly after, the intervention alerted him again to call on another girl for help. This time, she pointed to his screen and sounded out each syllable before she tapped it and completed the word. She continued this behavior, sounding out each syllable before tapping it on his tablet for several questions, and then returned to her own work.

Students also got much better at providing quality help and monitoring their cognition over time. We use the term "cognition monitoring" to characterize students metacognition when they needed help regardless of the system's suggestions. At the beginning of the experiment, most students followed the system suggestions by calling their peers for help, but helpers dismissed most requests. As the sessions progressed, students became increasingly aware of when and from whom they needed help and ignored the system suggestions when they deemed it inappropriate. Although the number of requests reduced with more experience with the intervention (Table 1), students started to call for help when they felt they actually needed some assistance, and not just because the system instructed them to. Figure 6 shows the percentage of positive help interactions (interactions where help was actually provided) broken down by classes over the six sessions.

In session 1 and most of session 2, helpers dismissed all help requests but started providing better help starting in session 3. Even though we became more explicit about demonstrating effective help interactions, students who needed help got much better at insisting that their peers actually help them when called. In all treatment groups (except for those in N1), we observed several incidents where students called helpers back who had originally dismissed their requests, students complained when their request were repeatedly dismissed, and even started ignoring suggestions from the help-seeking system if it suggested a helper who typically did not assist when called upon. We also observed incidents where other students stepped in to help their peers if an assigned helper ignored or dismissed the help request.

Finally, we found that students in groups with higher overall pretest scores were more likely to provide help for their peers rather than dismiss help requests. We ran a linear regression to determine if the average group pretest score had an impact on the percentage of positive help interactions within sessions (Table 3). The results 


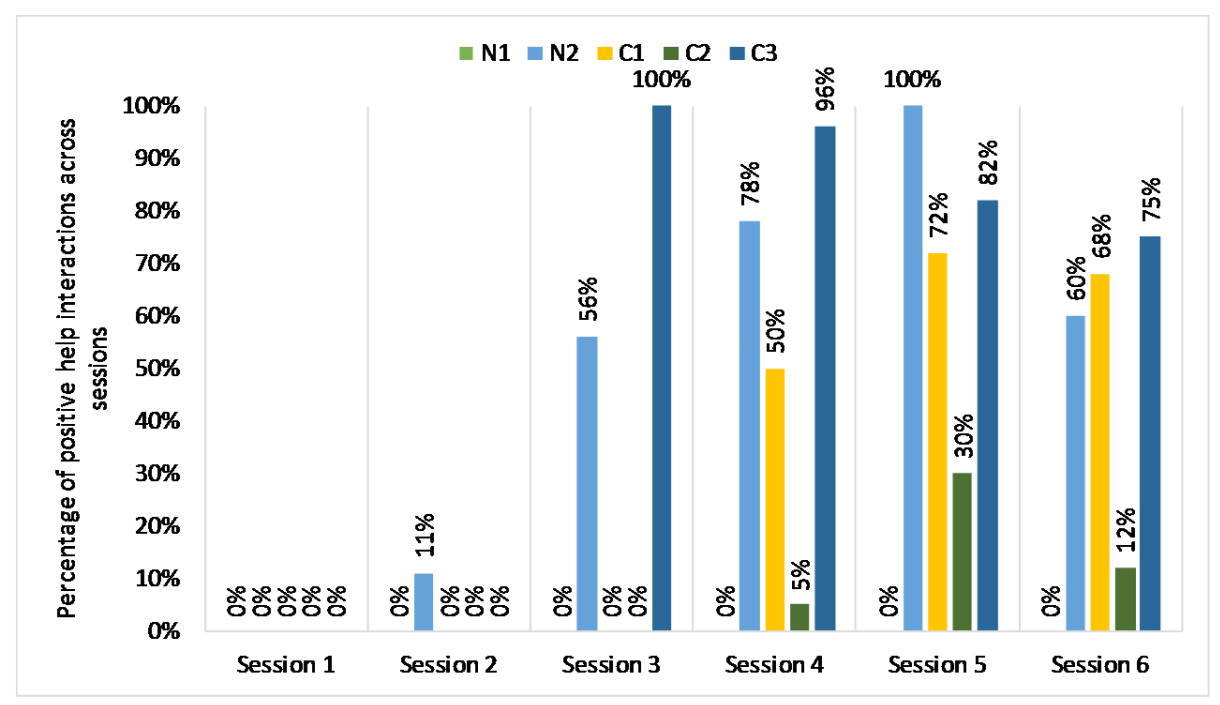

Figure 6: Percentage of positive help interactions across sessions for all classes

Table 3: Linear Regression results analyzing the effects of group pretest scores, and repeated exposure on positive help interactions (outcome variable)

\begin{tabular}{|c|c|c|c|c|c|}
\hline & Estimate & Std. Error & t value & $\operatorname{Pr}(>|t|)$ & sig \\
\hline Intercept & -2.50 & 2.11 & -1.19 & 0.240603 & \multirow{5}{*}{ * } \\
\hline Group Pretest Score & 0.08 & 0.04 & 2.15 & 0.035532 & \\
\hline Session Number - 1 & -0.05 & 1.90 & -0.03 & 0.979191 & \\
\hline Session Number - 2 & 0.39 & 1.90 & 0.21 & 0.835829 & \\
\hline Session Number - 3 & 1.62 & 1.90 & 0.85 & 0.39738 & \\
\hline Session Number - 4 & 6.73 & 1.90 & 3.55 & 0.000785 & *** \\
\hline Session Number - 5 & 5.19 & 1.96 & 2.65 & 0.010331 & * \\
\hline Session Number - 6 & 4.73 & 1.90 & 2.49 & 0.01558 & * \\
\hline
\end{tabular}

show that for every additional point in the groups average pretest score, there is an $8 \%$ significant increase in the number of positive help interactions when students are called upon in the group. The effects of the group pretest scores were magnified exponentially as students had more exposure to help-seeking intervention in additional sessions (as shown in the estimates from sessions 4-6).

Overall, results from this subsection show some promise that with proper training, careful group composition, and technology scaffolding, these young students can support their peers in ways bring about longer term learning of the material for both the helpers and the beneficiaries.

\subsection{H-2: System Engagement}

4.3.1 Intervention Makes No Difference In Student Activity Completion Rates. To measure student engagement, we analyzed the system logs to determine if students in the treatment condition completed activities at rates different from the control groups. We ran a linear regression model to determine if the treatment condition and the class significantly impact student activity completion rates (Table 4). The results show that overall, there was no significant difference in the activity completion rates between the treatment and the control groups.

Although it is unclear why the intervention had no effect on activity completion rates despite the availability of additional help to complete activities, one possible explanation based on insights from our previous study [63] is that for our participants and similar demographics, activity completion may be determined by how much students enjoy activities rather than their ability or the support they have for completing them. Therefore, our hypothesis H2-a is not proven - students in the treatment group do not complete activities at higher rates.

4.3.2 Intervention Causes Students To Select More Difficult Activities. Our learning system was divided into three main content areas Math, Literacy, and Stories. The 'Math' and 'Literacy' content areas contained graded activities, while the 'Stories' content area mostly required students to passively listen to ungraded, grade appropriate, and culturally relevant stories. The easiest activities in our system were contained in the "Stories" area - students mostly listened to the stories and invited their friends to share interesting bits. Therefore, students content area selection was not only reflective of the material they wanted to engage in, but also how much effort 
Table 4: Linear regression model on the effects on the system intervention and student classes on activity completion rates (outcome variable)

\begin{tabular}{|c|c|c|c|c|c|}
\hline & Estimate & Std. Error & $\mathrm{t}$ value & $\operatorname{Pr}(>|t|)$ & Sig \\
\hline Class - N1 Control Condition (Intercept) & 0.43 & 0.02 & 17.59 & $<2 \mathrm{e}-16$ & *** \\
\hline System Intervention & -0.01 & 0.02 & -0.54 & 0.587 & \\
\hline Class - N2 & 0.04 & 0.03 & 1.43 & 0.153 & \\
\hline Class - P1 & 0.03 & 0.03 & 0.94 & 0.347 & \\
\hline Class - P2 & 0.04 & 0.03 & 1.49 & 0.136 & \\
\hline Class - P3 & 0.05 & 0.03 & 1.49 & 0.137 & \\
\hline
\end{tabular}

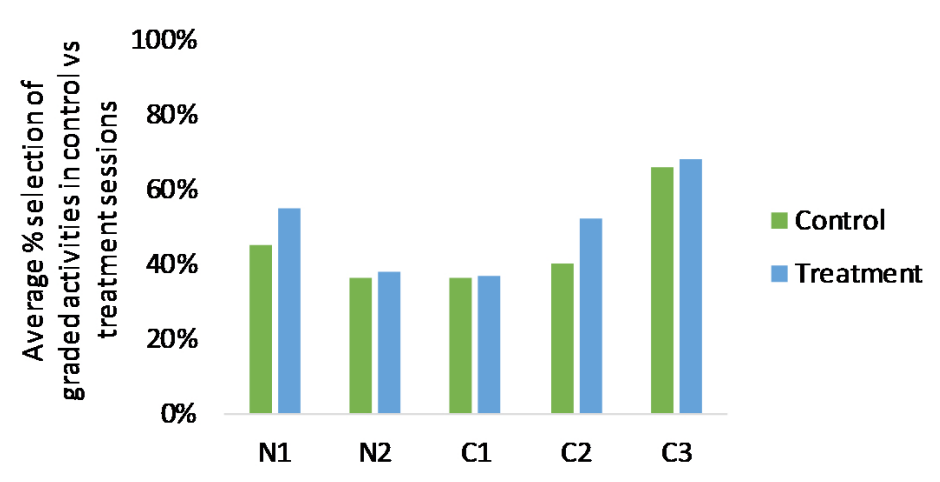

Figure 7: Activity Selection of graded activities in Control vs Treatment Groups.

Table 5: Linear Regression Model to analyze differences between Student Math/Literacy vs Stories Content Selection

\begin{tabular}{lccccc}
\hline & Estimate & Std. Error & t value & $\operatorname{Pr}(>|t|)$ & Sig \\
\hline Control Condition (Intercept) & 0.44217 & 0.01448 & 30.544 & $<2 \mathrm{e}-16$ & $* * *$ \\
Treatment Condition & 0.04893 & 0.02018 & 2.424 & 0.0156 & $*$ \\
\hline
\end{tabular}

they were willing to put into their learning at different points in the sessions. Figure 7 shows that for every grade (including N1 students who did not follow any of the intervention suggestions), the treatment group selected graded activities at a higher rate than the students in the control groups. Table 14 shows the results of a linear regression to determine the statistical difference in students content selection choices - the results shows that students in the treatment group selected graded activities an average of $4 \%$ more than the control group. These results are statistically significant even after we controlled for individual groups.

We were surprised by these results especially for N1 as they did not use the intervention in the way that we originally intended. However, all students were excited to see their faces on the tablets regardless of their use of the intervention. For the Nursery students, the possibility of seeing their faces on the tablet likely caused them to select the math/literacy activities more, and as a result, engaged in more cognitively challenging activities. For the other students, the guarantee that someone will be available to assist them if they ran into any trouble likely caused this behavior. These results show that our hypothesis $\mathrm{H} 2-b$ is true - the intervention causes students to select harder activities within the learning system.

\subsection{H-3: Learning Gains}

4.4.1 Students Score Similarly Within The Learning System. Within the learning system, we hypothesized that students in the treatment condition will outperform their peers in the control groups because they had assistance with any application and knowledge related issues they encountered. We ran a linear regression model (Table 6) to understand the significance of these differences - the results show that the system intervention had no statistically significant effects on student learning. As expected, students in higher grades performed better than students in Nursery 1 as they had higher domain knowledge overall. Given that the number of help requests related to knowledge support were so few compared to the other support types during the experiment, it is no surprise that overall, students in the control group did not perform better than those in the treatment groups. However, we observed that students in $\mathrm{P} 2$ gained less with our learning system compared to students in lower grades (N2 and P1), therefore, we reviewed video footage from those sessions to investigate why.

There was a literacy learning application (SPELL) that prompted P2 students to call for help too frequently. This application was experienced by students in P1, P2, and C3, but most frequently by 
Table 6: Linear Regression Model - Effect of System Intervention on student Average scores (outcome variable) in Math and Literacy Activities

\begin{tabular}{|c|c|c|c|c|c|}
\hline & Estimate & Std. Error & $\mathrm{t}$ value & $\operatorname{Pr}(>|t|)$ & Sig \\
\hline Class - N1 (Intercept) & 0.554987 & 0.030185 & 18.386 & $<2 \mathrm{e}-16$ & $* * *$ \\
\hline System Intervention & 0.003452 & 0.022657 & 0.152 & 0.878966 & \\
\hline Class - N2 & 0.214882 & 0.036502 & 5.887 & $<0.01$ & $* * *$ \\
\hline Class - P1 & 0.229663 & 0.038152 & 6.02 & $<0.01$ & $* * *$ \\
\hline Class - P2 & 0.141115 & 0.036551 & 3.861 & $<0.01$ & $* * *$ \\
\hline Class - P3 & 0.268961 & 0.037746 & 7.126 & $<0.01$ & $* * *$ \\
\hline
\end{tabular}

P2. The system prompted students to call for help before most of them got the chance to answer the questions on their own. We had very limited usage data on this SPELL application from our prior studies, therefore the timings were insufficient to prompt students appropriately. We observed students quickly get fatigued from moving around too often while learning with this application. Out of frustration, most students in P2 switched to the 'Stories' content area (and prompted their friends to switch as well) for the rest of the sessions, spending much less time in graded Math and Literacy activities. These results show that without reliable application timing data on the individual activities, the intervention prompting students too often and unnecessarily can have detrimental effects on student performance. Our analysis shows that the hypothesis $\mathrm{H} 3-\mathrm{a}$ is not true, students in the treatment condition did not perform better than those in the control condition on activities within the learning system. To better evaluate the hypothesis, we must resolve all application timing related issues and gather the data over a longer period to determine the intervention's effect on student learning within the system.

\section{DISCUSSION}

Our research study provides valuable insights on the benefits of incorporating technology-mediated help-seeking scaffolding into the practice of young students learning with technology unassisted in rural low-resourced areas. It also provides critical insights that such a system can be detrimental to students learning if not executed properly. To begin, our help-seeking intervention fostered knowledge support interactions that were not observed in any of the control groups. These interactions are the most critical of all the support types we tried to cater for, as it is traditionally the primary responsibility of teachers. In unassisted learning situations, students need additional support when they cannot attain new knowledge independently. Previous studies in this cultural context such as [65] have shown that without proper scaffolding, students have difficulty providing this support for one another, and an intervention such as ours can help students become better at it. We show evidence that students became aware that the help-seeking system was a tool that allowed them find support when they needed it students repeatedly tapped (or their peers directed them to tap) on the intervention when they needed help even when the system did not detect any struggles. This type of capability not only gives students the agency to request support when they need it, but also allows students to choose whether they want to accept or reject the suggestions without publicly informing other members of the group.

Our intervention also propelled students to engage in more cognitively challenging activities. For the youngest students, this might have been due to the excitement of seeing their faces on the tablets. For their older counterparts, they may have had increased confidence and comfort knowing that they had support if they ran into trouble. This insight has implications for how educators and researchers organize learning content especially for activities that students do not traditionally enjoy. As our data shows, students are more likely to gravitate to activities they enjoy most, or those that are less cognitively tasking. This is not at all unique to students in Tanzania - several studies have shown similar results for students from other demographics [14, 16, 33]. Designing engaging and enjoyable activities should be a primary goal for educators, learning scientists, and technology designers, however, it is sometimes difficult to balance student engagement with maximizing learning goals. It is especially difficult to design activities that are engaging for demographics such as our study participants where there is limited existing research on. Researchers often use technology as a probe to investigate activities that students find the most engaging [33] but that can be unreliable when technology itself is foreign to the target demographic. As researchers spend more time investigating ways to design activities that maximize both student engagement and learning, interventions such as our help-seeking system can allow students to further engage with existing learning activities as more engaging ones are being developed.

An indirect but highly beneficial aspect of our help-seeking system is that it allowed for better self-monitoring of students' cognition. As students in our treatment condition spent more time with our help-seeking intervention they got much better at only calling for help when they actually needed it, regularly insisting that their peers help them if they were dismissed and used the intervention to call on particular students depending on the type of help they needed. Students in the control groups (and in our previous two studies with this demographic) did not exhibit this level of agency and control on understanding when they needed help and who they needed it from. Although we did not find any studies directly from Tanzania (or even Africa), there is some research on interventions that specifically boost self-regulation and metacognitive skills in preschool to elementary school aged children e.g. [71]. Existing strategies that have shown positive results for improving students self-cognition include training kindergarten teachers about selfregulation e.g. in [45]. Meichenbaum et al. [30] found that primary 
school students with teachers who used strategies such as thinkalouds, reciprocal teaching, and cooperative learning showed better monitoring of their own cognition while learning. While most research conducted on this topic focus on implementing strategies in a traditional classroom environment, our research shows that technology-based interventions that employ similar strategies (our system fosters reciprocal teaching and cooperative learning) can also produce similar benefits. Longer term studies of such interventions are required to determine if these skills lead to improved learning gains on traditional tests.

Our system intervention also granted students what we might term "social permission", that is, the feeling of freedom to request for help from specific students while learning with the technology while reducing the face threat of making such a request. Students could have very easily turned around and called on any of their peers for help, but they relied on the system for permission to seek help in an environment where they were not mandated to accept the system recommendations unlike peer assignments from a teacher. Even more than giving students permission, our system provided them with knowledge of the specific peers that could help resolve their issues, providing them knowledge about students' ability that they would have not been aware of otherwise. Incorporating such technologies in and out of the classroom can potentially make students aware of their own abilities (in addition to their peers) in ways that encourage them to answer more questions in the classroom. Such awareness is not only relevant for students, but also teachers as well. Providing teachers with data on how students abilities evolve, as well as a wider range of the types of support that students can provide is likely to lead to a more equitable distribution of responsibilities in learning groups in classrooms, and reduce the anxieties that lower achieving students may face that prevent them from speaking up when called upon.

For personalized learning systems such as ours where content is adjusted to students' knowledge levels, it may seem redundant to design an intervention that fosters peer-peer knowledge support for students. However, our study shows that although these requests occur infrequently, they happen often enough that learning systems need to account and design for them. Students may also need additional support if a system focuses on testing and practice rather than actual teaching of educational concepts. In the short period of our research study, we did not see any differences in student learning based on the additional knowledge support, however, it is worth exploring over a longer period to determine how improvements to the system might affect student learning. There are also other metrics that can be assessed in future iterations of the intervention to explore other potential benefits of our system. For example, an increase in the percentage of positive help interactions for students in lower scoring groups might illustrate that the system is leading to increased confidence among lower achieving students on their abilities as helpers. Rather than just focusing on students' academic achievements, our future studies can employ interview and survey instruments validated in studies with young children to measure improvements to their metacognitive awareness (e.g. $[54,73])$, help-seeking and interpersonal cohesiveness (e.g. [7, 56]), and their collaborative skills (e.g. [20]) as a result of the intervention. Additionally, technological improvements can be made to our system by utilizing the camera and microphone to determine how long students spend, and how much discourse they engage in as a measure of the quality of help students provide.

Despite the potential benefits of such a system, our research shows that if not executed correctly, such a system can be detrimental to students learning even in a short period of time. Students in $\mathrm{C} 2$ gained less than their peers because they spent too much time in an application with inaccurate timing triggers, and the system prompted them to support their peers for issues that they could have overcome on their own. Students seemed to enjoy supporting one another in the treatment sessions, however, this should not have come at the expense of their learning gains. If these detrimental effects could have been observed even in the short term, they are potentially more devastating if students are not able to work around system issues on their own. It is critical that before such a system is deployed, researchers take the time to gather representative application timings to prevent reduced learning gains as we saw in our research study. Although we conducted three rounds of pilot testing with our help-seeking intervention (two in the United States, and one round with our target demographic), such problems were only observable over a large volume of users tested over a considerable amount of time.

Our system intervention shows some promise but requires improvements and further investigation to determine how beneficial it is for student learning. This research study gave us an opportunity to gather almost 700 hours of student usage logs and application timings - this is sufficient for improving the timing models for all our applications to prevent the errors encountered in our study. Improving application timing models is critical for avoiding application related errors (such as those from the SPELL application). While the calls for knowledge support did not show any benefits for student learning for the duration of our experiment, the productive nature of those interactions make it worthy of further investigation on a much longer-term study. Student should also be allowed to request for help from the system when they need it. We disabled this feature to prevent students from abusing the system, however, our data shows that students repeatedly tried to activate the intervention when they were genuinely struggling without success. Although students may abuse that feature due to excitement at first, this is likely to be normalized over time and used appropriately. The key factor to consider here is that students in our study demonstrated the need to maintain the agency of when they decided to call for support, and from whom.

\section{CONCLUSION}

We designed an Adaptive Collaborative Learning Support (ACLS) system specifically to address the needs of K-3 students in lowresource and rural communities, which does not rely on language comprehension for proper function. Like most ACLS, our system provided domain knowledge support, but additionally addresses struggles that students with low digital literacy are more likely to encounter including support for touchscreen gestures, and helping students overcome application errors unrelated to domain content. Our system was also designed to foster equitable help-seeking in group learning settings rather than pre-assigned tutor-tutee student pairs in most ACLS systems, empowering both high and low achieving students. We conducted a mixed-methods experimental 
study with 98 K-3 students in a rural village in Tanzania evaluating the system's effectiveness for improving student interactions, system engagement, and overall learning. Our system significantly increased student interactions with one another, fostered knowledge support interactions that were not observed in any of the control groups, propelled students to engage in more cognitively challenging activities, allowed for better self-monitoring of students' cognition, and granted students the social permission to request for help from specific students while reducing the face threat of making such a request. Further refinements to the system are needed to determine the long-term effects of such an intervention on student learning.

\section{ACKNOWLEDGMENTS}

We wish to thank our collaborators in Tanzania who have continuously welcomed us into their communities and provided valuable insights that have improved our learning system. We thank all the translators and native Swahili speakers who have ensured that we have translated content and context appropriately across the different media forms. We also wish to thank the Global Learning XPRIZE Initiative for providing us with the funding and resources to conduct such a study. We thank the Jacobs Foundation for their continued support for our research. Finally, we thank everyone who has contributed to this project by providing software solutions, technical support, domain knowledge support and consulting on our learning content. The research reported here was supported in part by the Institute of Education Sciences, U.S. Department of Education, through grant R305B150008 to Carnegie Mellon University. The opinions expressed are those of the authors and do not represent the views of the Institute or the U.S. Department of Education.

\section{REFERENCES}

[1] Philip C. Abrami*, Catherine Poulsen, and Bette Chambers. 2004. Teacher motivation to implement an educational innovation: Factors differentiating users and non-users of cooperative learning. Educational Psychology 24, 2: 201-216.

[2] Enrique Alfonseca, Rosa M. Carro, Estefanía Martín, Alvaro Ortigosa, and Pedro Paredes. 2006. The impact of learning styles on student grouping for collaborative learning: a case study. User Modeling and User-Adapted Interaction 16, 3-4: 377 401.

[3] Margarita Azmitia. 1996. Peer interactive minds: Developmental, theoretical, and methodological issues. Interactive minds: Life-span perspectives on the social foundation of cognition: 133-162.

[4] Nilufar Baghaei, Antonija Mitrovic, and Warwick Irwin. 2007. Supporting collaborative learning and problem-solving in a constraint-based CSCL environment for UML class diagrams. International fournal of Computer-Supported Collaborative Learning 2, 2-3: 159-190.

[5] Stephany Berinstein and Lilian Magalhaes. 2009. A study of the essence of play experience to children living in Zanzibar, Tanzania. Occupational therapy international 16, 2: 89-106.

[6] Cynthia Breazeal, Robin Morris, Stephanie Gottwald, Tinsley Galyean, and Maryanne Wolf. 2016. Mobile devices for early literacy intervention and research with global reach. In Proceedings of the Third (2016) ACM Conference on Learning@Scale, 11-20.

[7] William M. Bukowski, Betsy Hoza, and Michel Boivin. 1994. Measuring friendship quality during pre-and early adolescence: The development and psychometric properties of the Friendship Qualities Scale. Fournal of social and Personal Relationships 11, 3: 471-484.

[8] Tak-Wai Chan and Chih-Yueh Chou. 1997. Exploring the design of computer supports for reciprocal tutoring.

[9] Xinyin Chen, Kenneth H. Rubin, and Dan Li. 1997. Relation between academic achievement and social adjustment: Evidence from Chinese children. Developmental psychology 33, 3: 518.

[10] Cosmas Cobbold. 2006. Attracting and retaining rural teachers in Ghana: The premise and promise of a district sponsorship scheme. Fournal of Education for Teaching 32, 4: 453-469.
[11] B. A. K. Cocroft and Stella Ting-Toomey. 1994. Facework in Japan and the United States. International fournal of Intercultural Relations 18, 4: 469-506.

[12] Juliet Corbin and Anselm Strauss. 2008. Basics of qualitative research: Techniques and procedures for developing grounded theory. Thousand Oaks.

[13] Don Dansereau and D. W. Johnson. 1994. Cooperative learning. Learning, remembering, believing: Enhancing human performance: 83-111.

[14] Andrea DeBruin-Parecki. 2009. Establishing a family literacy program with a focus on interactive reading: The role of research and accountability. Early Childhood Education fournal 36, 5: 385-392.

[15] Janice Webb Early. 1999. The impact of peer tutoring on self-esteem and Texas Assessment of Academic Skills mathematics performance of tenth-grade students.

[16] B. Egeland, N. Weinfield, M. Hiester, C. Lawrence, S. Pierce, K. Chippendale, and J. Powell. 1995. Teaching tasks administration and scoring manual. University of Minnesota.

[17] Ruth Evans. 2012. Sibling caringscapes: Time-space practices of caring within youth-headed households in Tanzania and Uganda. Geoforum 43, 4: 824-835.

[18] Charles R. Greenwood. 1996. Research on the practices and behavior of effective teachers at the Juniper Gardens Children's Project: Implications for the education of diverse learners. Research on classroom ecologies: 39-67.

[19] Willard W. Hartup. 1992. Having Friends, Making Friends, and Keeping Friends: Relationships as Educational Contexts. ERIC Digest.

[20] Danielle Herro, Cassie Quigley, Jessica Andrews, and Girlie Delacruz. 2017. CoMeasure: developing an assessment for student collaboration in STEAM activities. International journal of STEM education 4, 1: 26.

[21] John M. Hintze, Robert J. Volpe, and Edward S. Shapiro. 2002. Best practices in the systematic direct observation of student behavior. Best practices in school psychology 4: 993-1006.

[22] M. Gail Jones and Glenda Carter. 1994. Verbal and nonverbal behavior of abilitygrouped dyads. Journal of Research in Science Teaching 31, 6: 603-619.

[23] Alison King, Anne Staffieri, and Anne Adelgais. 1998. Mutual peer tutoring: Effects of structuring tutorial interaction to scaffold peer learning. Fournal of Educational Psychology 90, 1: 134 .

[24] T. Kuleana. 1999. The State of Education in Tanzania: crisis and opportunity. Kuleana Centre, Dar es Salaam.

[25] Rohit Kumar, Carolyn Penstein Rosé, Yi-Chia Wang, Mahesh Joshi, and Allen Robinson. 2007. Tutorial dialogue as adaptive collaborative learning support. Frontiers in artificial intelligence and applications 158: 383.

[26] Robert A. LeVine, Suzanne Dixon, Sarah LeVine, Amy Richman, Constance H. Keefer, P. Herbert Leiderman, and T. Berry Brazelton. 1996. Child care and culture: Lessons from Africa. Cambridge University Press.

[27] Marlaine E. Lockheed and Adriaan M. Verspoor. 1991. Improving primary education in developing countries. Oxford University Press for World Bank.

[28] Gift Masaiti and Pamela Nachona Naluyele. 2011. Strategies to retain and motivate employees in Africa: Examining the case of the ministry of education in Zambia. African Journal of Political Science and International Relations 5, 8: 409-423.

[29] Ruth Meena. 2018. Situational analysis of education of girls/women in Tanzania. Utafiti fournal 3, 2.

[30] Donald Meichenbaum and Andrew Biemiller. 1998. Nurturing independent learners: Helping students take charge of their learning. ERIC.

[31] Laura Menikoff. 1999. The effects of cross-age tutoring upon the decoding skills, attitude toward reading, teacher perceptions of reading improvement, and the self-concept of inner-city at-risk students. City University of New York.

[32] Sugata Mitra, Ritu Dangwal, Shiffon Chatterjee, Swati Jha, Ravinder S. Bisht, and Preeti Kapur. 2005. Acquisition of computing literacy on shared public computers: Children and the" hole in the wall". Australasian fournal of Educational Technology 21,3 .

[33] Amelia K. Moody, Laura M. Justice, and Sonia Q. Cabell. 2010. Electronic versus traditional storybooks: Relative influence on preschool children's engagement and communication. Journal of Early Childhood Literacy 10, 3: 294-313.

[34] Emma Msaki. 2006. THE UNITED REPUBLIC OF TANZANIA MINISTRY OF EDUCATION AND VOCATIONAL TRAINING Education Sector Development Programme. Retrieved August 12, 2019 from https://www.academia.edu/7040480/THE_UNITED_REPUBLIC_OF_ TANZANIA_MINISTRY_OF_EDUCATION_AND_VOCATIONAL_TRAINING_ Education_Sector_Development_Programme

[35] Lyabwene Mtahabwa and Nirmala Rao. 2010. Pre-primary education in Tanzania: Observations from urban and rural classrooms. International fournal of Educational Development 30, 3: 227-235.

[36] Aidan Mulkeen and Dandan Chen. 2008. Teachers for rural schools: experiences in Lesotho, Malawi, Mozambique, Tanzania, and Uganda. The World Bank.

[37] P. G. Nalkur and Achievement Orientations. 2009. Strategies: A Cultural Comparison of Tanzanian Street Children, Former Street Children, and School-Going Children. Fournal of Cross-Cultural Psychology 40, 6: 1014.

[38] Joe D. Nichols and Raymond B. Miller. 1994. Cooperative learning and student motivation. Contemporary Educational Psychology 19, 2: 167-178.

[39] Aannemarie Sullivan Palinscar and Ann L. Brown. 1984. Reciprocal teaching of comprehension-fostering and comprehension-monitoring activities. Cognition and instruction 1, 2: 117-175. 
[40] Judy M. Parr and Michael AR Townsend. 2002. Environments, processes, and mechanisms in peer learning. International journal of educational research 37, 5 : 403-423.

[41] Souren Paul, Priya Seetharaman, Imad Samarah, and Peter P. Mykytyn. 2004 Impact of heterogeneity and collaborative conflict management style on the performance of synchronous global virtual teams. Information \& Management 41, 3: 303-321.

[42] Daniel Pauw, Tamara Clegg, June Ahn, Elizabeth Bonsignore, Jason C. Yip, and Judith Uchidiuno. 2015. Navigating connected inquiry learning with ScienceKit. . International Society of the Learning Sciences, Inc.[ISLS].

[43] Roy D. Pea. 1993. Practices of distributed intelligence and designs for education Distributed cognitions: Psychological and educational considerations 11: 47-87.

[44] Roy D. Pea. 2004. The social and technological dimensions of scaffolding and related theoretical concepts for learning, education, and human activity. The journal of the learning sciences 13, 3: 423-451.

[45] Nancy E. Perry. 1998. Young children's self-regulated learning and contexts that support it. fournal of Educational Psychology 90, 4: 715-729. https://doi.org/10. 1037/0022-0663.90.4.715

[46] Nguyen Phuong-Mai, Cees Terlouw, and Albert Pilot. 2005. Cooperative learning vs Confucian heritage culture's collectivism: Confrontation to reveal some cultural conflicts and mismatch. Asia Europe fournal 3, 3: 403-419. https://doi.org/ 10.1007/s10308-005-0008-4

[47] Liana Razmerita and Armelle Brun. 2011. Collaborative learning in heterogeneous classes. In The 3rd International Conference on Computer Supported Education 189194.

[48] Amanda J. Rose and Steven R. Asher. 2004. Children's strategies and goals in response to help-giving and help-seeking tasks within a friendship. Child Development 75, 3: 749-763.

[49] Carolyn Rosé, Yi-Chia Wang, Yue Cui, Jaime Arguello, Karsten Stegmann, Armin Weinberger, and Frank Fischer. 2008. Analyzing collaborative learning processes automatically: Exploiting the advances of computational linguistics in computersupported collaborative learning. International journal of computer-supported collaborative learning 3, 3: 237-271.

[50] Nikol Rummel, Erin Walker, and Vincent Aleven. 2016. Different futures of adap tive collaborative learning support. International fournal of Artificial Intelligence in Education 26, 2: 784-795.

[51] Lawrence Sherman. 2000. SOCIOMETRY IN THE CLASSROOM. Retrieved September 9, 2019 from http://www.users.miamioh.edu/shermalw/sociometryfiles/ sociocl.htmlx

[52] Georg Siemens. 2005. Connectivism: A learning theory for the digital age. International Journal of Instructional Technology and Distance Learning. Obtained through the Internet: http://www.idtl.org/Journal/Jam_05/article01.htm. [Ac cessed Sept. 2008].

[53] Robert E. Slavin. 1983. When does cooperative learning increase student achievement? Psychological bulletin 94, 3: 429.

[54] Rayne A. Sperling, Bruce C. Howard, Lee Ann Miller, and Cheryl Murphy. 2002 Measures of children's knowledge and regulation of cognition. Contemporary educational psychology 27, 1: 51-79.

[55] Sandra J. Stone and James F. Christie. 1996. Collaborative literacy learning during sociodramatic play in a multiage $(\mathrm{K}-2)$ primary classroom. fournal of Research in Childhood Education 10, 2: 123-133.

[56] Sarah Strohkorb, Ethan Fukuto, Natalie Warren, Charles Taylor, Bobby Berry, and Brian Scassellati. 2016. Improving human-human collaboration between children with a social robot. In 2016 25th IEEE International Symposium on Robo and Human Interactive Communication (RO-MAN), 551-556.
[57] Anna Every Swai. 2013. The Effects of Incentive Initiatives on Teacher Retention in Tanzania: A Case of the Rukwa Region.

[58] Peter Taylor and Abigail Mulhall. 2001. Linking learning environments through agricultural experience-enhancing the learning process in rural primary schools. International fournal of Educational Development 21, 2: 135-148.

[59] Patricia Azevedo Tedesco. 2003. MArCo: Building an artificial conflict mediator to support group planning interactions. International fournal of Artificial Intelligence in Education 13, 1: 117-155.

[60] Stella Ting-Toomey. 1988. Rhetorical sensitivity style in three cultures: France, Japan, and the United States. Central States Speech fournal 39, 1: 28-36. https: //doi.org/10.1080/10510978809363232

[61] Stella Ting-Toomey. 1988. Intercultural conflict styles: a face-negotiation theory. In Theories in intercultural communication, Y. Kim and W. Gudykunst (eds.). SAGE, Newbury Park, CA, 213-235.

[62] A. Tsui. 1996. Reticence and anxiety in second language teaching. In Voices from the language classroom, K. Bailey and D. Nunan (eds.). Cambridge University Press, Cambridge, 145-167.

[63] Judith Uchidiuno, Evelyn Yarzebinski, Emily Keebler, Kenneth Koedinger, and Amy Ogan. 2019. Learning from african classroom pedagogy to increase student engagement in education technologies. In Proceedings of the Conference on Computing \& Sustainable Societies, 99-110.

[64] Judith Uchidiuno, Evelyn Yarzebinski, Michael Madaio, Nupur Maheshwari, Ken Koedinger, and Amy Ogan. 2018. Designing Appropriate Learning Technologies for School vs Home Settings in Tanzanian Rural Villages. In Proceedings of the 1st ACM SIGCAS Conference on Computing and Sustainable Societies, 9.

[65] Judith Uchidiuno, Evelyn Yarzebinski, Emilio Vargas-Vite, Ken Koedinger, and Amy Ogan. 2019. The Effectiveness of Publicly vs. Privately Assigned Group Leaders among Learners in Rural Villages in Tanzania.

[66] Ema Ushioda. 1996. Learner autonomy 5: The role of motivation. Authentik.

[67] Ana Cláudia Vieira, Lamartine Teixeira, Aline Timóteo, Patrícia Tedesco, and Flávia Barros. 2004. Analyzing on-line collaborative dialogues: The oxentchêchat. In International Conference on Intelligent Tutoring Systems, 315-324.

[68] Erin Walker, Nikol Rummel, and Kenneth R. Koedinger. 2014. Adaptive intelligent support to improve peer tutoring in algebra. International fournal of Artificial Intelligence in Education 24, 1: 33-61.

[69] Felix Warneken, Jasmin Steinwender, Katharina Hamann, and Michael Tomasello. 2014. Young children's planning in a collaborative problem-solving task. Cognitive Development 31: 48-58.

[70] Paula Michele White. 2000. Promoting mathematics achievement, academic efficacy, and cognitive development of at-risk adolescents through deliberate psychological education. University of Houston.

[71] David Whitebread, Penny Coltman, Deborah Pino Pasternak, Claire Sangster, Valeska Grau, Sue Bingham, Qais Almeqdad, and Demetra Demetriou. 2009. The development of two observational tools for assessing metacognition and self-regulated learning in young children. Metacognition and learning 4, 1: 63-85.

[72] L Xiao. 2006. Bridging the gap between teaching styles and learning styles: a cross-cultural perspective. Teaching English as a Second or Foreign Language 10, 3: $1-15$.

[73] Barry J. Zimmerman and Dale H. Schunk. 2011. Self-regulated learning and performance. Handbook of self-regulation of learning and performance: 1-12.

[74] 2013. Teacher Allocation and Absenteeism in East Africa:: KiuFunza:: Twaweza.org. Retrieved August 29, 2019 from https://www.twaweza.org/go/ teacher-absenteeism-in-east-africa

[75] Rethinking languages of instruction in African schools | Development Education Review. Retrieved August 6, 2020 from https: //www.developmenteducationreview.com/issue/issue-4/rethinking-languagesinstruction-african-schools 